\title{
PIEZOELECTRIC ACTUATORS FOR BONE MECHANICAL STIMULATION: EXPLORING THE CONCEPT
}

\author{
Joana Reis (1), Clara Frias (2), Fernando Capela-Silva (3), José Potes (3), Luísa Botelho \\ (4), Carlos Castro (4), António Marques (5), José Simões (6) \\ 1.Universidade de Évora (UÉ), Portugal; 2. INEGI, Portugal; 3. UÉ-ICAAM, Portugal; \\ 4.ITN, Portugal; 5.FEUP, Portugal; 6. Universidade de Aveiro, Portugal
}

\section{Introduction}

Arthroplasty is liable to cause intense changes on strain levels and distribution in the bone surrounding the implant, namely stress shielding. Several solutions have been proposed for this, namely design variations and development of controlled-stiffness implants (Simões, 2000). A new approach to this problem, with potential application to other orthopaedic problems and other medical fields, would be the development of smart implants integrating systems for bone mechanical stimulation. Ideally, the implant should present sensing capability and the ability to maintain physiological levels of strain at the implant interface. Piezoelectric materials' huge potential as a mean to produce direct mechanical stimulation lies on the possibility of producing stimuli at a high range of frequencies and in multiple combinations. The present in vitro and preliminary in vivo studies were a first step towards the validation of the concept.

\section{Methods}

Polymeric piezoelectric films [Polyvinylidene Fluoride (PVDF)] with silver electrodes were after dip-coated to improve cell adhesion and assure electrical insulation as described elsewhere (Frias et al., 2010). These films were used as substrate for MC3T3 cell growth in static-control and dynamic conditions. In dynamic conditions the substrates were deformed by applying a $5 \mathrm{~V}$ current, at $1 \mathrm{~Hz}$ and $3 \mathrm{~Hz}$ for 15 minutes. Cell proliferation and viability and nitric oxide (NO) production were assessed and immunofluorescence studies on the cytoskeleton performed.

For the in vivo study, an actuator device was developed, composed microcontroller (Texas Instruments, U.S.A) and a set of 6 actuators identical to the ones used in in vitro and using the same dynamic regimen. A similar, but static, control device was also developed. The actuator and control static devices were implanted in the hind limbs of a 4 year old merino ewe. The 6 actuators and static controls were placed inside 6 osteotomy slots cut into the proximal tibial and distal femoral physis. 30 days after implantation, the animal was sacrificed and the biological response around the osteotomies was assessed through histology and histomorphometry in nondecalcified sections and histochemistry and immunohistochemistry in decalcified sections. The present study conducted accordingly to FELASA's guidelines for animal care.

\section{Results}

Cell viability was significantly decreased in the groups grown on the device surface although higher in the group subjected to stimulation. NO was significantly higher in the dynamic group. Immunofluorescence evidenced stronger fluorescence on mechanical stimulated cells and prominent cytoplasmatic extensions.

In the in vivo study total bone area around the actuators was significantly higher, when comparing to static controls $(39.91 \pm 14.08 \%$ vs. $27.20 \pm 11.98 \%$ ). The increment of bone occupied area was due to new bone formation. In actuators the area occupied by woven bone and osteoid was $64.89 \pm 19.32 \%$ of the total bone area $v s$. $31.72 \pm 14.54 \%$ in static devices. Bone deposition rate was significantly higher around actuated devices than around static devices Immunohistochemistry shown a marked elevation in osteopontin detection around actuators when compared to static controls. An increase in osteocalcin was also observed.

\section{Discussion}

The present results suggest that piezoelectric materials and the converse piezoelectric effect may be used to effectively stimulate bone growth. In vitro measurements of $\mathrm{NO}$ also suggest osteoblasts detect and respond in a reproducible way to small displacements and strain levels. The development of fully biocompatible devices based on this concept represents both a massive challenge and a wealth of possible applications with high impact on the biomedical field and on knowledge on cell mechanotransduction mechanisms.

\section{References}

Frias et al. J Biomech 43: 1061-1066, 2010.

Simões et al. Compos Sci Technol 60: 559-567, 2000. 